\title{
Performance evaluation of a simple decoding method for millimeter-wave short- range MIMO transmission through a wall
}

\author{
Kazumitsu Sakamoto ${ }^{\text {a) }}$, Ken Hiraga, Tomohiro Seki, \\ Tadao Nakagawa, and Kazuhiro Uehara \\ NTT Network Innovation Laboratories, NTT Corporation \\ Hikarinooka 1-1, Yokosuka-shi, 239-0847 Japan \\ a)sakamoto.kazumitsu@lab.ntt.co.jp
}

\begin{abstract}
The simple decoding method we have proposed for shortrange multiple-input multiple-output (SR-MIMO) transmission is a promising means for reducing power consumption. The method performs MIMO detection with analog devices, thus reducing the number of quantization bits required in the analog-to-digital converter (ADC) of the receiver and the amount of signal processing calculation for MIMO detection. However, when the method is applied to a wall transmissive wireless repeater on a multilayered wall, the transmission performance degrades due to multipath generated by the multilayered structure. In this letter, we evaluate the method's performance using data for a millimeter wave propagation channel that we measured from wall samples and the measured S-parameters of the method's analog circuit. As a result, we quantify the influence of multipath generated by a wall's multilayered structure on transmission performance.
\end{abstract}

Keywords: short-range MIMO, millimeter-wave, wall transmissive wireless repeater, simple decoding method

Classification: Wireless Communication Technologies

\section{References}

[1] N. Honma, K. Nishimori, T. Seki, and M. Mizoguchi, "Short range MIMO communication," Proceedings of the 3rd European Conference on Antennas and Propagation (EuCAP 2009), pp. 1763-1767, March 2009.

[2] I. Sarris and A. R. Nix, "Design and performance assessment of highcapacity MIMO architectures in the presence of a line-of-sight component," IEEE Trans. Veh. Technol., vol. 56, no. 4, pp. 2194-2202, July 2007.

[3] T. Seki, K. Nishimori, K. Hiraga, and K. Nishikawa, "Experimental evaluation of high speed parallel data transmission technology for wireless repeater system," IEEE Radio and Wireless Symposium (RWS), pp. 304307, Jan. 2010. 
[4] R. Kataoka, K. Nishimori, K. Hiraga, K. Takai, K. Sakamoto, and T. Seki, "Simple decoding method for short range MIMO transmission," IEICE Technical Report, AP2011-173, pp. 153-158, Jan. 2012 (in Japanese).

[5] [Online] http://yoshino-gypsum.com/kouhou/taika/taika02.html.

[6] [Online] http://www.alc.gr.jp/only/fireproof.html.

[7] P. B. Kenington and L. Astier, "Power consumption of A/D converters for software radio applications," IEEE Trans. Veh. Technol., vol. 49, no. 2, pp. 643-650, March 2000.

[8] K. Nishimori, N. Honma, T. Seki, and K. Hiraga, "On the transmission method for short range MIMO communication," IEEE Trans. Veh. Technol., vol. 60, no. 3, pp. 1247-1251, March 2011.

[9] K. Sakamoto, K. Hiraga, T. Seki, T. Nakagawa, and K. Uehara, "Performance evaluation of short-range MIMO using a method for controlling phase difference between each propagation channel," IEICE Trans. Commun., vol. E96-B, no. 10, pp. 2513-2520, Oct. 2013.

[10] IEEE P802.11ad Draft Std, D2.0, March 2011.

[11] K. Sato, T. Manabe, T. Ihara, H. Saito, S. Ito, T. Tanaka, K. Sugai, N. Ohmi, Y. Murakami, M. Shibayama, Y. Konishi, and T. Kimura, "Measurements of reflection and transmission characteristics of interior structures of office building in the 60-GHz band," IEEE Trans. Antennas Propag., vol. 45, no. 12, pp. 1783-1792, Dec. 1997.

\section{Introduction}

In our research, we have focused on short-range multiple-input multipleoutput (SR-MIMO) transmission, which performs MIMO transmission by utilizing the length differences in the propagation channels between facing transmitting ( $\mathrm{Tx})$ and receiving $(\mathrm{Rx})$ array antenna elements in short range wireless communications [1,2]. An SR-MIMO transmission application we consider is a wall transmissive wireless repeater that is installed on both sides of a wall [3]. The repeater enables high speed data transmission to be performed through the wall from one room to another. We have proposed a simple decoding method [4] for SR-MIMO transmission that can perform MIMO detection with low power consumption. This method enables spatial multiplexed signals to be separated in radio frequency (RF) band with analog devices. When it is applied to a multilayered partition wall in an ordinary house $[5,6]$, reflected waves occurring at the boundary surface may pose a problem. Rx antenna elements receive not only direct waves but also delayed waves due to the reflection. Multipath degrades the simple decoding method's performance but the influence of multipath on the method has not been reported yet. In this letter, we evaluate the method's performance using data for a millimeter wave propagation channel that we measured from multilayered wall samples and the measured S-parameters of the method's analog circuit (hereafter "the simple decoding circuit"). As a result, we quantify the degree to which the multipath inside a wall degrades the performance. 


\section{SR-MIMO transmission through a wall}

\subsection{Simple decoding method for SR-MIMO}

When the number of $\mathrm{Tx}$ and $\mathrm{Rx}$ antenna elements is increased in case of general MIMO detection methods using digital signal processing, the number of quantization bits required in the analog-to-digital converter (ADC) of the receiver and the amount of signal processing calculation are increased, and as a result the power consumption is increased [7]. On the other hand, the simple decoding method [4] enables MIMO detection to be performed in RF band with an analog circuit. This solves the aforementioned problems so that MIMO detection with low power consumption can be expected. Figure 1 shows a $2 \times 2$ SR-MIMO transmission model using the simple decoding method, which has two Tx and two Rx antenna elements. Here, $h_{i j}$ ( $i$ and $j$ : positive integers less than or equal to 2 ) denotes the propagation channel component comprising the direct waves from the $j$-th Tx antenna element to the $i$-th Rx antenna element. In SR-MIMO transmission, there is an optimum array antenna's element spacing $d$ that maximizes the channel capacity for a certain transmission distance $D$ [8]. A $2 \times 2$ channel matrix with optimum element spacing is approximated by Eq. (1).

$$
\mathbf{H}=\left[\begin{array}{ll}
h_{11} & h_{12} \\
h_{21} & h_{22}
\end{array}\right] \simeq h_{11}\left[\begin{array}{cc}
1 & -\alpha j \\
-\alpha j & 1
\end{array}\right]
$$

where $\alpha$ is a real constant that depends on the radiation characteristic of the antenna element, the transmission distance, etc. $\alpha$ is defined by Eq. (2).

$$
\alpha=\left|\frac{h_{21}}{h_{11}}\right| .
$$

As shown in Eq. (1), the phase difference $\theta_{H}=\arg \left(h_{21} / h_{11}\right)=\arg \left(h_{12} / h_{22}\right)$ $=-90^{\circ}[9]$. In this case, the zero forcing (ZF) weight matrix for MIMO detection is approximated by Eq. (3).

$$
\mathbf{W}=\mathbf{H}^{-1} \simeq w_{11}\left[\begin{array}{cc}
1 & \alpha j \\
\alpha j & 1
\end{array}\right]
$$

As shown in Fig. 1, the weight matrix $\mathbf{W}$ is easily constructed using analog devices such as a 90-degree phase shifter, attenuator, and divider. In the attenuators, the signals are attenuated as a function of $\alpha$. When two signals

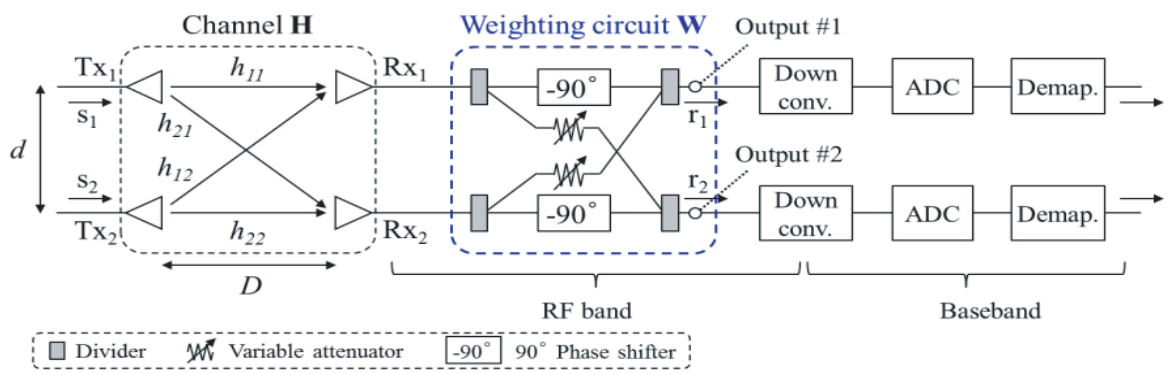

Fig. 1. A $2 \times 2$ SR-MIMO transmission model using the simple decoding method. 
transmitted by the transmitter are represented by the vector $\mathbf{S}=\left[\mathrm{s}_{1}, \mathrm{~s}_{2}\right]^{T}$, where $(.)^{T}$ denotes transpose, and additive white Gaussian noise (AWGN) at the receiver is represented by the vector $\mathbf{n}=\left[\mathrm{n}_{1}, \mathrm{n}_{2}\right]^{T}$, the received signal vector $\mathbf{R}=\left[\mathrm{r}_{1}, \mathrm{r}_{2}\right]^{T}$ output to the weighting circuit can be represented as Eq. (4).

$$
\mathbf{R}=\mathbf{W}(\mathbf{H S}+\mathbf{n}) .
$$

Substituting Eq. (1) and Eq. (3) into this equation confirms that full-rank MIMO transmission can be achieved.

$$
\begin{aligned}
\mathbf{R} & =\left[\begin{array}{cc}
1 & \alpha j \\
\alpha j & 1
\end{array}\right]\left[\begin{array}{cc}
1 & -\alpha j \\
-\alpha j & 1
\end{array}\right] \mathbf{S}+\mathbf{W n} \\
& =\left[\begin{array}{cc}
1+\alpha^{2} & 0 \\
0 & 1+\alpha^{2}
\end{array}\right] \mathbf{S}+\mathbf{W n} .
\end{aligned}
$$

\subsection{Influence of multipath generated inside a wall on simple decoding method's performance}

A wall transmissive wireless repeater we have proposed performs high speed data transmission through a wall from one room to another. However, a problem may occur when applying the simple decoding method to the repeater on a partition wall in an ordinary house, since such walls usually have a multilayered structure $[5,6]$. In such cases, when the signals transmitted by the Tx antenna elements of the repeater propagate toward the Rx antenna elements installed on the opposite side of the wall, some of the signals are transmitted through the boundary surfaces of the multiple layers and the others are reflected at the boundary surfaces. Thus, the Rx antenna elements receive direct waves that arrive after being transmitted through the layer's boundary surfaces and delayed waves that arrive while repeating the reflection and transmission occurring at the boundary surfaces. The combination of direct and delayed waves may cause amplitude and phase errors to occur in the optimum propagation channel (shown in Eq. (1)), and these errors would degrade the simple decoding method's MIMO signal separation performance. There is a possibility that the application of the SR-MIMO simple decoding method on the wall transmissive wireless repeater is difficult depending on the degree of the influence of the delayed waves.

\section{Performance evaluation}

\subsection{Measurement of MIMO channels through a wall}

Figure 2 shows the structures of the walls we used to measure the propagation channels. The layer structure and thickness are the same as those of a partition wall in an ordinary house [5, 6]. As shown in Fig. 2 (a), Plasterboard wall A comprises plasterboard (regular type, Yoshino Gypsum Co., Ltd.) layers of $12.5 \mathrm{~mm}$ thickness placed on either side of a layer of air and light-gauge steel (LGS) of $65.0 \mathrm{~mm}$ thickness. As shown in Fig. 2 (b), Plasterboard wall B comprises two plasterboard (regular type, Yoshino Gypsum Co., Ltd.) layers of $12.5 \mathrm{~mm}$ thickness placed on either side of a layer of air 


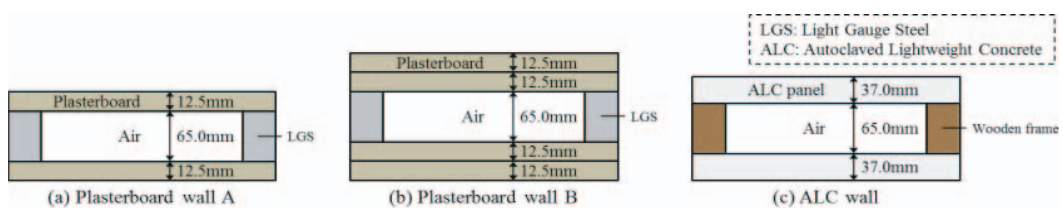

Fig. 2. Typical structures of a partition wall in an ordinary house.

and LGS of $65.0 \mathrm{~mm}$ thickness. As shown in Fig. 2 (c), the ALC wall comprises autoclaved lightweight concrete (ALC) panels (flat panel, Asahi Kasei Construction Materials Corp.) of $37.0 \mathrm{~mm}$ thickness placed on either side of a layer of air and wooden frames of $65.0 \mathrm{~mm}$ thickness. We installed horn antennas (SGH-15-RP000, Millitech Inc.) across these walls and then measured a $2 \times 2 \mathrm{MIMO}$ channel at $1.76 \mathrm{GHz}$ bandwidth and $62.64 \mathrm{GHz}$ center frequency with a network analyzer. The measurements were performed by installing the $\mathrm{Tx}$ and $\mathrm{Rx}$ antenna elements in a position where there were no wooden frames or LGS between them. For comparison, we also measured the propagation channel through air only, with Tx-to-Rx distance of $139 \mathrm{~mm}$ (the same as the ALC wall thickness). In each measurement (for Plasterboard wall A, Plasterboard wall B, ALC wall, and air), element spacing was adjusted so that the phase difference $\theta_{H}=-90^{\circ}$ at the center frequency.

\subsection{Performance evaluation using measured MIMO channel data}

We evaluated the $2 \times 2$ SR-MIMO transmission performance by computer simulation using the measured channel data and the measured S-parameters of the simple decoding circuit. The first modulation scheme is QPSK or 16 QAM or 64 QAM and the second is orthogonal frequency division multiplexing (OFDM). In accordance with IEEE 802.11ad standard [10], center frequency is $62.64 \mathrm{GHz}$, subcarrier spacing is $5.16 \mathrm{MHz}$, and the number of subcarrier is 336 . The S-parameters of the simple decoding circuit we used are the characteristics measured at the same relative bandwidth at $4.85 \mathrm{GHz}$ center frequency as a scale model of the millimeter-wave band. Figures 3 (a), (b) and (c) show the bit error rate (BER) performance of the simple decoding method. The target BER before error correction is less than $10^{-2}$ (BER $<10^{-2}$ ), which becomes error-free when low-density parity-check (LDPC) codes are used as error correction codes. As shown in Figs. 3 (a), (b) and (c), the required signal-to-noise ratio (SNR) for each first modulation scheme to satisfy BER $<10^{-2}$ for Plasterboard wall $\mathrm{A}$ is increased by $1 \mathrm{~dB}$ for QPSK and $2 \mathrm{~dB}$ for $16 \mathrm{QAM}$ as compared with the case of air only. We confirmed that the BER for $64 \mathrm{QAM}$ does not become less than $10^{-2}$ and found that the required SNR for Plasterboard wall B increases very little for QPSK and $16 \mathrm{QAM}$ and is increased by $1 \mathrm{~dB}$ for $64 \mathrm{QAM}$. Furthermore, the required SNR for the ALC wall is increased by $1 \mathrm{~dB}$ for QPSK and 16 QAM and by $5 \mathrm{~dB}$ for 64 QAM. Figure $3(\mathrm{~d})$ shows the cumulative distribution function $(\mathrm{CDF})$ of the signal-to-interference ratio (SIR) of the subcarriers for Plaster- 


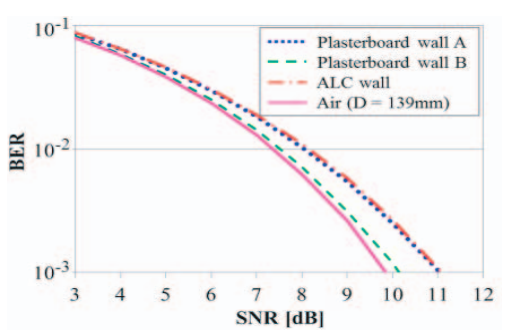

(a) BER performance (QPSK)

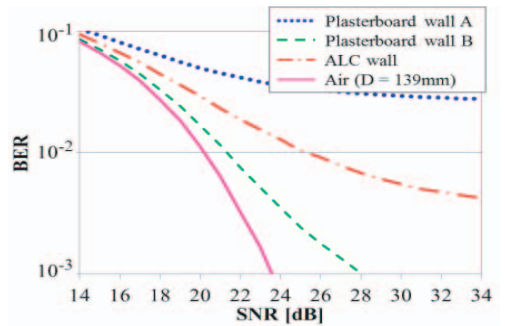

(c) BER performance (64QAM)

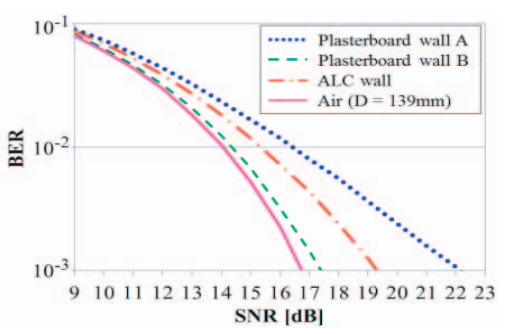

(b) BER performance (16QAM)

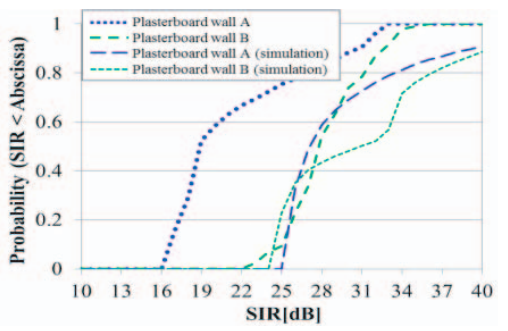

(d) CDF of the SIR of subcarriers

Fig. 3. BER performance and CDF of the SIR of subcarriers for the $2 \times 2$ SR-MIMO simple decoding method.

board wall A and B. The SIR is calculated by using the measured channel data and ideal simple decoding method's weight that does not have amplitude and phase errors to evaluate the influence of the channel. For comparison, the CDF of the SIR calculated by using a $2 \times 2$ MIMO channel generated by a ray-tracing method is also shown. We consider up to two reflections from the boundary surfaces. Plasterboard's dielectric constant used in simulation is $2.50-j 0.03$ [11]. Because the amplitude and phase errors of the propagation channel increase as the influence of multipath inside a wall increases, the desired signals are not combined in same phase at the output of the simple decoding circuit shown in Fig. 1 and the interference signals remain without being combined in opposite phase. As a result, SIR degrades. Comparing the CDF of the SIR for Plasterboard wall A with that for Plasterboard wall B, we can see that the proportion of subcarriers with high SIR is higher for Plasterboard wall B and the influence of multipath is smaller in both measurement and simulation. This is because the dielectric part made of plasterboard for Plasterboard wall B is thicker than that for Plasterboard wall $\mathrm{A}$ and therefore the power ratio of the direct waves to the delayed waves becomes larger. Plasterboard is a non-uniform material within which bubbles are contained. It is assumed that the SIR values in measurement are lower than the values in simulation because they are influenced by not only the reflected waves at the boundary surfaces which are considered in simulation but also the reflected waves from the bubbles.

\section{Conclusion}

Using the measured channel data and the measured S-parameters of the simple decoding circuit, we quantified the influence of multipath inside a wall, which are a factor in performance degradation when the SR-MIMO simple 
decoding method we proposed is applied to a wall transmissive wireless repeater on a multilayered wall. We measured MIMO channels for four different cases: two types of plasterboard walls (A and B), an ALC wall, and air only with no walls. For Plasterboard wall A we found that the required SNR to satisfy BER $<10^{-2}$ increases by $1 \mathrm{~dB}$ for QPSK and by $2 \mathrm{~dB}$ for 16 QAM as compared with the case of air only, and that the BER for 64 QAM does not become less than $10^{-2}$. For Plasterboard wall B we found that the required SNR increases very little for QPSK and 16 QAM and by $1 \mathrm{~dB}$ for 64 QAM. For the ALC wall we found that the required SNR increases by $1 \mathrm{~dB}$ for QPSK and 16 QAM and by $5 \mathrm{~dB}$ for 64 QAM.

\section{Acknowledgments}

The authors would like to thank Associate Prof. Dr. Kentaro Nishimori at Niigata University for the useful advice and information he provided to us in discussions. 